\title{
The importance of gut microbiota in pregnancy: prevention, care and support
}

\begin{abstract}
The microbiota i.e. all those good bacteria along our intestines, influencing the maturation of the immune system, affects our health and well-being and protect us from diseases such as allergies, diabetes and even cancer. That's why we often hear talk about the microbiota. It all starts with the birth, (eutocico) and during vaginal passage, begins the exchange of bacteria that will colonize the intestines of the unborn child and be able to educate the immune system to recognize what belongs all body (self), from what is foreign (non-self). It will speak, therefore, tolerance of immunological, this phenomenon will continue with the breast feeding and the exchange with the saliva of the mother. We can say that the microbiota of the mother, closely related to food choices, it is essential for proper immune development of their child, then why not pay attention all the nutrition during pregnancy?
\end{abstract}

Volume 4 Issue 3 - 2016

Diana Yedid

Dyd Integrative Medicine, Italy

Correspondence: Diana Yedid, Dyd Integrative Medicine,Via Carpaccio, I2 20133 Milano, Italy, Tel 3933934854II, Email dianayedid599@gmail.com

Received: November 17, 2015 | Published: March 3, 2016

\section{Introduction}

It resides in the human gut ecosystem most densely populated of microorganisms that carry out essential functions in the homeostasis of the immune system in the metabolism of the host. The community of intestinal microorganisms (bacteria, fungi, protozoa and viruses) are collectively called "microbiota" and with all their genomes "microbiome". ${ }^{1}$ The intestinal microbiota is continuously balanced with the gut-associated lymphoid tissue (gut-associated lymphoid tissue, GALT), the largest and most important immune organ of the human body. 2

Immunological homeostasis is the result of a continuous crosstalk between the microbiota and the mucosal immune system. The mucosal immune system is part of both the innate and adaptive, immune response. The host takes great advantages immunologically and metabolically by physical proximity between the gut microbial populations and underlying tissues, but at the same time this proximity is a continuing threat to health. In fact, the immune system must establish the right balance between "tolerance" to the intestinal microbiota, keeping sty- inflammatory tone, and "vigilance" with regard to infectious agents and opportunistic pathogens. 3 The intestinal microbiota is also essential for the digestive activity and this action involves bacterial species that reside mainly in the colon. These microbiota promote breakdown, by fermentation, of Soluble fiber, disaccharides and peptides that escape digestion in the small intestine.

This fermentation produces, among other substances, short-chain fatty acid such as acetic acid, propionic acid and butyric acid. Shortchain fatty acids help to control fluid balance, as they are the primary energy source for colonocytes. Short-chain fatty acids improve the absorption of minerals and positively influence the metabolism of lipids and glucose in the liver.

The evolution of the microbial intestine begins at birth, ends during the first years of life and is highly customizable and stable in a healthy individual. ${ }^{3}$ At birth the intestinal tract is sterile, but soon develops a microbiota whose composition varies depending on factors such as the type of childbirth, nutrition during childhood, antibiotic use and diet, and then complete its maturation by the $3 \mathrm{rd}-4$ th year of life. ${ }^{4}$ Acute or chronic disturbance of equilibrium of these intestinal microorganisms is therefore an important factor in many chronic diseases such as human inflammatory bowel disease, obesity, type 2 diabetes and cancer of the colon and rectum. This alteration called dysbiosis may be favored not only by an improper diet, use of antibiotics, stress and alterations of the sleep-veglia, but also chronic diseases that produce portal hypertension or alter the micro-level of the basal lamina of the gastro-intestinal tract contribute to this situation.

As a result of these perturbations, the number of bacteria such as lactobacilli and bifidobacteria decreases to the advantage of pathogenic species such as some clostridia and enterococci, ${ }^{5}$ but above all increases bacterial translocation generating an increased nonspecific immunological response.

The intestinal microbiota (1013-1014 cells/intestinal contents) is the most complex ecosystem found in nature that is home to several hundred species of bacteria. These bacteria belong to the phylum Bacteroidetes, Firmicutes, Proteobacteria, and Actinobacteria Fusobacteria. This microbiota is commonly referred to as our hidden metabolic 'organ' due to their immense impact on human wellbeing, including host metabolism, physiology, nutrition and immune function. These communities, characterized by metabolic specialization, complementarity and cooperation, are a very complex network of interaction microbe-microbe and microbe-host (intestinal brain).

The interaction between host and microbiota previously defined in a reductive as diner (only one of the two benefits), is actually very active, symbiotic or mutualistic, where the immunity system is the most involved in this report, especially in maintaining the balance between tolerance and immune activation.

The immunological surveillance of the populations of intestinal microorganisms involves receptor proteins belong to the class of pattern recognition receptors (PPR), which recognize the structural reasons preserved expressed by microorganisms, pathogen associated molecular patterns (PAMP) through the toll- like receptors (toll-like receptors, TLRs) and nucleotide binding oligomerization domain (NOD). These receptors act in distinct cellular compartments thus contributing to tolerance towards commensal bacteria and food-borne antigens. 6 The recognition of these receptors on the apical surface of the epithelium promotes tolerance and the right tone inflammatory; on the contrary the activation of these same receptors from the side basolateral promotes strong inflammatory responses. 
Cooperative as well as competitive interactions may occur between the microbiota, via 'pattern recognition receptors' (TLRs and NODs), or other components of the innate immune system leading to proinflammatory or anti-inflammatory immune responses in different cell types.

The intestinal microflora plays a definitely important role both in intestinal inflammation that in the development of tumors of the gastro-intestinal tract. Alterations of this ecosystem have been observed such as in older people with cancer of the colon and rectum. In particular, it has been highlighted the prevalence of clostridia at the expense of bifidobacteria compared to healthy elderly in these individuals. ${ }^{7}$ The interaction between the microbiota and the immune system in cancer patients is important not only for the aspects of the pathogenesis but also because it can provide an opportunity for therapeutic management/ frequency of surgical complications is related to the assumption of chemotherapy (lower incidence of secondary complications).

\section{Using soluble fractions of dietary fiber as immunological adjuvants}

The $\beta$-glucans are linear polysaccharides consisting of glucose molecules joined together by glycosidic bonds. ${ }^{8}$ Some types of betaglucan are destroyed by the gastric juices and thus are not orally active, but the beta fraction 1,3-1,6-D-glucan, derived from baker's yeast, is stable. The receptors Members of the recognition of the beta-1, 3-1.6 D-glucan are expressed by macrophages and thus able to induce the activation. In a study conducted by Bogwald \& collaborators, ${ }^{9}$ it was shown that activated macrophages from glucan are able to recognize and phagocytose different types of cancer cells such as melanoma and mast cell tumor, while other researchers have shown that the glucan is capable of significantly reduce the growth of breast cancer cells and B16 melanoma in mouse models, suggesting that the glucan possesses also antitumor activity. ${ }^{10,11}$

The beneficial properties of beta-glucans in the supportive care of cancer are now well defined and clearly associated with their immunomodulatory activity. 8 It 'also known, that the combination of beta-glucans with other natural compounds can potentiate the activity. In particular, the acid ascorbic acid (vitamin C) is an important factor in the processes of macrophage activation. In fact, the activated macrophage consuming and requires ascorbic acid in amounts to 1,000 times higher than those present in the blood. More recent studies have shown that some bioactive molecules, such as vitamin $\mathrm{C}$ and resveratrol can further boost the impact of beta-glucan, when given in combination. ${ }^{12,13}$ It was observed that their combination suppresses more effectively the growth of mammary tumors and lung, promoting apoptosis of the cancerous cells. In this regard, some polysaccharides such as the $\beta$-Glucan, can be used not only for their ability to act as immunostimulants but also as prebiotics. Indeed, the $\beta$-glucans are highly fermented by the gut microbiota blind and the small intestine and can increase the rate of growth and production of lactic acid of microbes isolated human intestine. ${ }^{14}$ These findings could open up new therapeutic prospects integration using appropriate strains of probiotics combined with micronutrients ( $\beta-$ Glucan, Vit. C, resveratrol) in maintaining a healthy intestinal environment (eubiosis) and for a proper balance of the innate immune system.

\section{Conclusion}

Despite the composition and characteristics of an intestinal microbiota "healthy" are not yet well defined, it is known that perturbations of the composition of the microflora of the gastrointestinal tract are associated with an increased risk of immunemediated diseases. ${ }^{15}$ The growing interest in the effects of the intestinal microbiota in human health has prompted the development of strategies to optimize the microbial ecosystem and stressed the importance of nutrition and lifestyle in maintaining the abundance, variety and diversity of the intestinal microflora. Rebalancing a state of dysbiosis is still a complex operation because the microbial populations that have most influence immunological homeostasis are anaerobic bacteria which can hardly be built from the outside, through the use of biomodulator (probiotics, prebiotics, synbiotics, postbiotics). Therefore, acquiring a healthy lifestyle and a diet that can provide the body with several active substances, it is essential not only to reduce the risk of immune-mediated diseases in adults but also to promote a good immunological maturation in early life (core microbiota).

\section{References}

1. Icaza Chavez ME. Gut microbiota in health and disease. Rev Gastr Mex. 2013;78(4):240-248.

2. Pearson C, Uhlig HH, Powrie F. Lymphoid microenvironments and innate lymphoid cells in the gut. Trends Immunol. 2012;33(6):289-296.

3. Peterson CT, Sharma V, Elmén L, et al. Immune homeostasis, dysbiosis and therapeutic modulation of the gut microbiota. Clin Exp Immunol. 2015;179(3):363-377.

4. Salzman NH. The role of the microbiome in immune cell development. Ann Allergy Asthma Immunol. 2014:113(6):593-598.

5. Dejea CM, Wick EC, Hechenbleikner EM, et al. Microbiota organization is a distinct feature of proximal colorectal cancers. Proc Natl Acad Sci U S A. 2014;111(51):18321-18326.

6. Cario E. Bacterial interactions with cells of the intestinal mucosa: Tolllike receptors and NOD2. Gut. 2005;54(8):1182-1193.

7. Gao Z, Guo B, Gao R, et al. Microbiota disbiosis is associated with colorectal cancer. Front Microb. 2015;6:20.

8. Vannucci L, Krizan J, Sima P, et al. Immunostimulatory properties and antitumor activities of glucans. Int J Oncol. 2013;43(2):357-364.

9. Bogwald J, Johnson E, Seljelid R. The Cytotoxic Effect of Mouse Macrophages Stimulated in vitro by a $\beta-1,3-\mathrm{d}-$ Glucan from Yeast Cell Walls. Scandinavian Journal of Immunology. 1982;15(3):297-304.

10. Di Luzio NR, McNamee RB, Williams DL, et al. Glucan induced inhibition of tumor growth and enhancement of survival in a variety of transplantable and spontaneous murine tumor models. Adv Exp Med BioI. 1979;121(A):269-290.

11. Seljelid RA. Water-soluble aminated beta-i, 3D-glu-can derivative causes regression of solid tumors in mice. Biosci Rep Sett. 1986;6(9):845-851.

12. Vetvicka V, Vetvickova J. Combination of glucan, resveratrol and vitamin C demonstrates strong anti-tumor potential. Anticancer Res. 2012;32(1):81-87.

13. Vetvicka V, Volny T, Saraswat-Ohri S, et al. Glucan and resveratrol complex, possible synergistic effects on immune system. Biom Pap Med Fac Univ Palacky Olom Czech Rep. 2007;151(1):41-46.

14. Blaut M. Relationship of prebiotics and food to intestinal microflora. Eur J Nutr. 2002;41Suppl.1:i11-i16.

15. Annalisa N, Alessio T, Claudette TD, et al. Gut microbioma population: an indicator really sensible to any change in age, diet, metabolic syndrome, and life-style. Mediators Inflamm. 2014:901308. 